\title{
Vigilancia de la salud pública después de una erupción volcánica: lecciones aprendidas en Cerro Negro, Nicaragua, $1992^{1}$
}

\author{
Josephine Malilay ${ }^{2}$, Mariana Guido Real, ${ }^{3}$ \\ Álvaro Ramírez Vanegas, ${ }^{4}$ Eric Noji ${ }^{2}$ y Thomas Sinks ${ }^{2}$
}

RESUMEN La erupción del volcán Cerro Negro cerca de León, Nicaragua, el 9 de abril de 1992 lanzó alrededor de 1,7 millones de toneladas de ceniza en una zona de $200 \mathrm{~km}^{2}$. Se efectuó una evaluación inicial de los efectos en la salud de cerca de 300000 residentes, con los datos normalmente obtenidos mediante el sistema nacional de vigilancia epidemiológica. Se determinó que el número de consultas a los establecimientos de atención de salud por diarrea e infecciones respiratorias agudas (IRA) aumentó en las dos comunidades estudiadas, una dentro de la zona del desastre y otra en sus cercanías. En particular, las consultas por diarrea aguda fueron casi 6,0 veces más numerosas que antes de la erupción en ambas comunidades y las consultas por IRA, 3,6 veces más frecuentes en Malpaisillo (la comunidad cercana a la zona del desastre) y 6,0 veces más frecuentes en Telica (la comunidad situada dentro de esa zona). Casi todas fueron consultas por enfermedad en lactantes y niños menores de 5 años.

El aumento de la tasa de morbilidad por diarrea, que frecuentemente se produce después de las erupciones volcánicas, exige que se investiguen detalladamente el tipo y la calidad del abastecimiento de agua después de una acumulación cuantiosa de ceniza. Es preciso examinar con mayor detenimiento los problemas respiratorios asociados con la ceniza para determinar el espectro de esas enfermedades y el momento en que se presentan en lactantes y otros subgrupos especiales de la población. Los datos acopiados por medio de la vigilancia pasiva sobre las condiciones de salud antes y después de una erupción pueden emplearse para detectar la morbilidad relacionada con la erupción. Los sistemas que ya están establecidos, como el sistema nacional de vigilancia epidemiológica de Nicaragua, se pueden modificar o ampliar para mejorar su sensibilidad a nuevos casos y, por ende, su capacidad de ofrecer servicios de notificación apropiados a los organismos de socorro médico.

1 Este trabajo fue financiado por acuerdo contractual [APO-43482 (RR)] con el Programa de Preparativos para Situaciones de Emergencia y Coordinación del Socorro en Casos de Desastre de la Organización Panamericana de la Salud, Washington, DC, EUA. Se publicó en inglés en el Bulletin of the Pan American Health Organization, Vol. 30, No. 3, 1996, con el título "Public health surveillance after a volcanic eruption: lessons from Cerro Negro, Nicaragua, 1992".

2 División de Peligros Ambientales y Efectos en la Salud, Centro Nacional de Salud Ambiental, Cen- tros para el Control y la Prevención de Enfermedades, Atlanta, Georgia, EUA. Las solicitudes de separatas u otra correspondencia deberán dirigirse a Josephine Malilay a la siguiente dirección postal: Health Studies Branch (F-46), Division of Environmental Hazards and Health Effects, National Center for Environmental Health, Centers for Disease Control and Prevention, Atlanta, GA 30341-3724, EUA. Teléfono: 770-488-7350; fax: 770488-7335.

3 Ministerio de Salud, León, Nicaragua.

4 Ministerio de Salud, Managua, Nicaragua.
En investigaciones sobre los efectos que ejercen en la salud los desastres con consecuencias agudas ha habido una tendencia a emplear sistemas de vigilancia activos, es decir, sistemas en que se solicita información activamente, y no sistemas pasivos (los habituales) en los que esto no se hace (1-6). Tales sistemas activos pueden permitir 
la evaluación inmediata de los problemas vinculados con el desastre a fin de montar operaciones de socorro (7). No obstante, en el trabajo aquí notificado se ha empleado un sistema pasivo, el sistema nacional de vigilancia epidemiológica de Nicaragua, para detectar los efectos en la salud de una erupción volcánica y señalar algunos problemas cuya resolución podría reducir la morbilidad asociada con los peligros que presentan los volcanes. Los resultados obtenidos sirvieron para demostrar que, con algunas modificaciones, los sistemas habituales de información de salud, como el sistema nacional de vigilancia que aquí se usó, pueden proporcionar estimaciones razonables de los problemas sanitarios que pueden surgir después de un desastre.

\section{ANTECEDENTES}

El volcán Cerro Negro en Nicaragua $\left(12^{\circ} 51^{\prime}\right.$ de latitud norte y $86^{\circ} 70^{\prime}$ de longitud oeste), que está localizado unos $25 \mathrm{~km}$ al noroeste de León, capital del departamento del mismo nombre, entró en erupción a las 23.30 horas del 9 de abril de 1992 (8). En el curso de los 3 días siguientes, un chorro de lava depositó cerca de 1,7 millones de toneladas de ceniza en dirección oestesuroeste en una zona de $200 \mathrm{~km}^{2}$ que se extiende más allá de León y se sitúa unos $80 \mathrm{~km}$ al noroeste de Managua (figura 1). Al principio, las autoridades de salud locales expresaron su alarma ante los peligros de los gases tóxicos, ya que se notificaron concentraciones de dióxido de azufre nueve veces más altas de lo normal. Aunque no hubo víctimas, quedaron expuestos a riesgo 300000 habitantes de la zona afectada, de los cuales más de 10000 necesitaron algún tipo de ayuda de emergencia $(9,10)$.

\section{MATERIALES Y MÉTODOS}

Las listas semanales de las enfermedades notificables y defunciones correspondientes al mes anterior a la erupción se obtuvieron del Informe diario de vigilancia epidemiológica, vehículo

FIGURA 1. Mapa que indica los niveles de acumulación de ceniza tras la erupción del volcán Cerro Negro el 9 de abril de 1992, la altura del territorio circundante y la localización de León, capital del departamento del mismo nombre; Malpaisillo y Telica, las dos comunidades estudiadas; y otros pueblos vecinos y volcanes de las cercanías, indicados con una $\mathrm{V}$

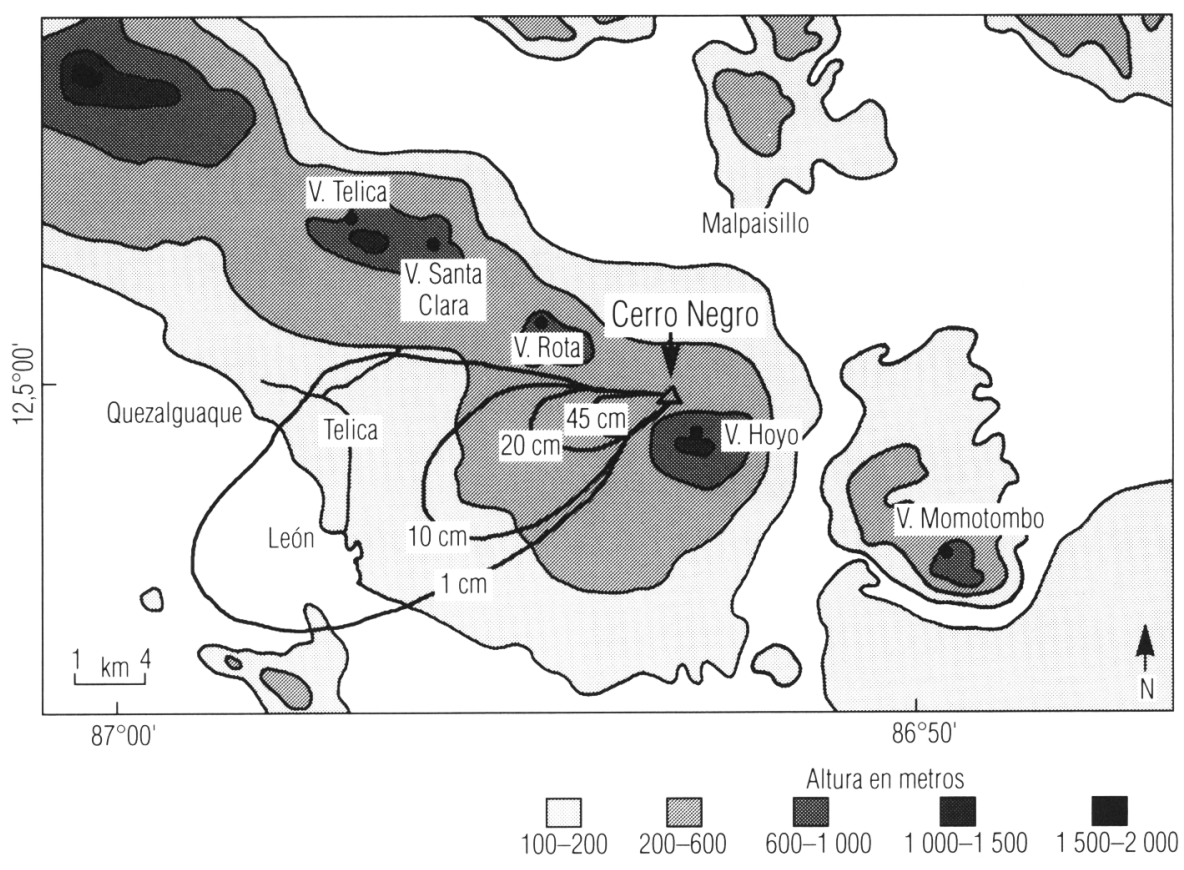

Fuente: Instituto Nicaragüense de Estudios Territoriales.

de notificación del sistema local de atención integral en salud (SILAIS), que es la unidad de notificación de información de salud del departamento de León. De la misma fuente de información se obtuvieron las enfermedades y defunciones notificadas durante la semana posterior a la erupción.

Nueve municipios del departamento más la ciudad de León notificaban a diario las estadísticas de morbilidad y mortalidad de sus respectivos hospitales, dispensarios y puestos de salud. Se seleccionaron dos de estas 10 subdivisiones que proporcionaban sus datos -Malpaisillo y Telica (con 35692 y 22378 habitantes, respectivamente) - , las cuales abarcaban alrededor de $48 \%$ del territorio departamental y contenían la mayor parte de la población que vivía fuera de la ciudad de León. Se dispuso de datos sobre la estructura de edad de la población de esos dos municipios (11).

La morbilidad por diarrea e IRA fue la única que se investigó en esas comunidades, ya que solo se observaron casos esporádicos de otras afecciones (tales como conjuntivitis, sarampión y casos de cólera sospechados) durante el período de estudio. Para fines de la investigación se examinaron los casos que, según los informes, habían ocurrido durante el período de notificación epidemiológica del 8 de marzo al 4 de abril de 1992 (antes de la erupción del 9 de abril) y los notificados entre el 12 y el 18 de abril, después de la erupción. El sistema de vigilancia no proporcionó ninguna definición de casos de diarrea aguda ni de IRA.

\section{RESULTADOS}

Las consultas semanales a servicios de atención de salud por diarrea 
aguda fueron mayores en niños menores de 1 año, grupo en que la tasa de consulta aumentó de 13,8 por 1000 niños durante el mes que precedió a la erupción a 45,1 por 1000 una semana después de ella. En términos de edad, el mayor número de consultas semanales, después del citado, se observó en niños de 1 a 4 años, en quienes la tasa aumentó de 2,3 por 1000 durante el mes anterior a la erupción a 23,2 por 1000 en la semana posterior. En la comunidad de Malpaisillo el número semanal de consultas a servicios de atención de salud por diarrea tendió a ser mucho mayor que en Telica (figura 2).

Antes de la erupción, las tasas semanales de consultas médicas por IRA eran mayores en Malpaisillo que en Telica. Dichas tasas mostraron aumentos notables en ambas comunidades después de la erupción, y en Telica aumentaron más de cuatro veces. Durante el período de estudio, los niños menores de 1 año (en particular) y los menores de 5 años invariablemente presentaron mayores tasas de IRA que los otros grupos de edad en ambas comunidades. En Malpaisillo las consultas sanitarias por IRA en lactantes mostraron una tasa que osciló entre 27,7 por 1000 cuatro semanas

FIGURA 2. Notificaciones semanales de consultas a servicios de salud por diarrea aguda por 1000 niños menores de 1 año y menores de 5 años en los municipios de Malpaisillo y Telica, Nicaragua, del 8 de marzo al 18 de abril de 1992

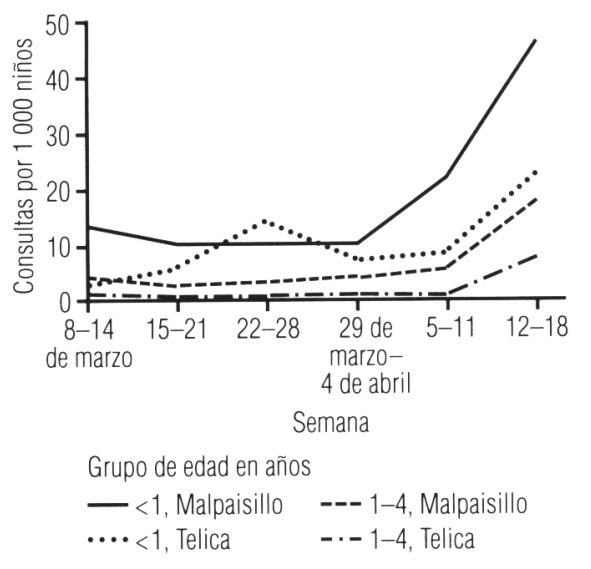

antes de la erupción y 79,9 por 1000 una semana después. En Telica, esas tasas variaron entre 17,4 por 1000 cuatro semanas antes y 83,6 por 1000 una semana después (figura 3).

Se comparó la morbilidad por diarrea e IRA antes y después de la erupción para determinar qué proporción del incremento de la tasa de morbilidad se relacionaba con la erupción. Los cuadros 1 y 2 muestran la tasa de morbilidad estandarizada (TME) y los intervalos de confianza (IC) de 95\% correspondientes a diarrea e infecciones respiratorias agudas en las comunidades estudiadas (12). Al tomar como estándar la tasa de morbilidad de cada grupo de edad en cada localidad antes de la erupción, se encontró que después de esta la morbilidad por diarrea aguda en ambas comunidades era 5,8 veces mayor de la anticipada. Asimismo, la morbilidad observada por IRA después de la erupción fue 3,6 veces mayor de la esperada en Malpaisillo y 6,0 veces mayor en Telica.

La morbilidad por diarrea aguda observada en lactantes fue aproximadamente 3 a 4 veces mayor después de la erupción en las dos comunidades estudiadas. Sin embargo, la morbilidad observada en niños de 1 a 4 años fue 5,9 veces mayor después de la erupción en Malpaisillo (IC95\%: 4,3 a $8,2)$ y 17,3 veces mayor en Telica (IC95\%: 7,1 a 42,1). En los otros grupos de edad, el riesgo relativo de sufrir diarrea aguda fluctuó entre 10 y 30 en Malpaisillo y 3 y 7 en Telica. En general, el riesgo aparente de diarrea aguda de todos los grupos de edad, salvo el de niños de 1 a 4 años, fue mayor en Malpaisillo que en Telica.

En comparación con la morbilidad registrada antes de la erupción, el aparente riesgo relativo de sufrir IRA después de ella fue de 3,6 en Malpaisillo y de 6,0 en Telica. Entre los grupos de edad específicos que fueron estudiados (cuadro 2), la morbilidad observada por IRA aumentó por un factor que varió de 2 a 10. Los riesgos relativos de contraer una IRA después de la erupción, comparados con los que había antes de ella, fueron invariablemente mayores en todos los grupos de edad en Telica que en los grupos
FIGURA 3. Notificaciones semanales de consultas a servicios de salud por infecciones respiratorias agudas por cada 1000 niños menores de 1 año y menores de 5 años en los municipios de Malpaisillo y Telica, Nicaragua, del 8 de marzo al 18 de abril de 1992

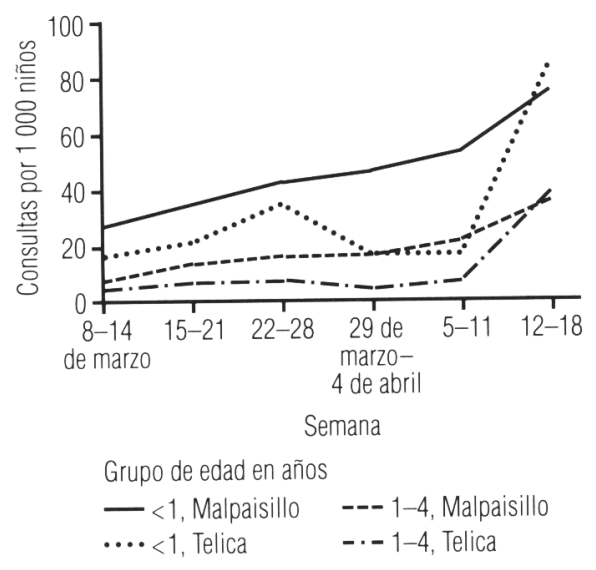

homólogos en Malpaisillo y variaron de 3,7 (IC95\%: 2,7 a 5,0) a 10,0 (IC95\%: 3,1 a 31,9). También detectamos casos de diarrea aguda, IRA y conjuntivitis en los habitantes de asentamientos de evacuación, pero no se observó ninguna tendencia que llamara la atención en la morbilidad diaria durante los 10 días posteriores a la erupción.

\section{DISCUSIÓN}

La vigilancia epidemiológica en los dispensarios de salud municipales reveló que las tasas semanales de consultas de salud por diarrea e IRA aumentaron después de la erupción, particularmente en niños menores de 4 años. Estas tasas semanales proporcionaron solo una aproximación a la incidencia de morbilidad, ya que es posible que algunos casos de enfermedad no hayan sido motivo de consulta y de que se hayan producido consultas repetidas a los establecimientos de atención de salud incluidos en el estudio.

Pese a que los residentes fueron evacuados de las zonas afectadas, el número de consultas a servicios de atención de salud aumentó durante la semana posterior a la erupción. Como las personas evacuadas aún no habían 
CUADRO 1. Casos de diarrea aguda en personas atendidas en los establecimientos de salud de Malpaisillo y Telica antes y después de la erupción, junto con las tasas de morbilidad estandarizadas (TME) y los intervalos de confianza de $95 \%$ (IC $95 \%$ ) derivados de esos datos

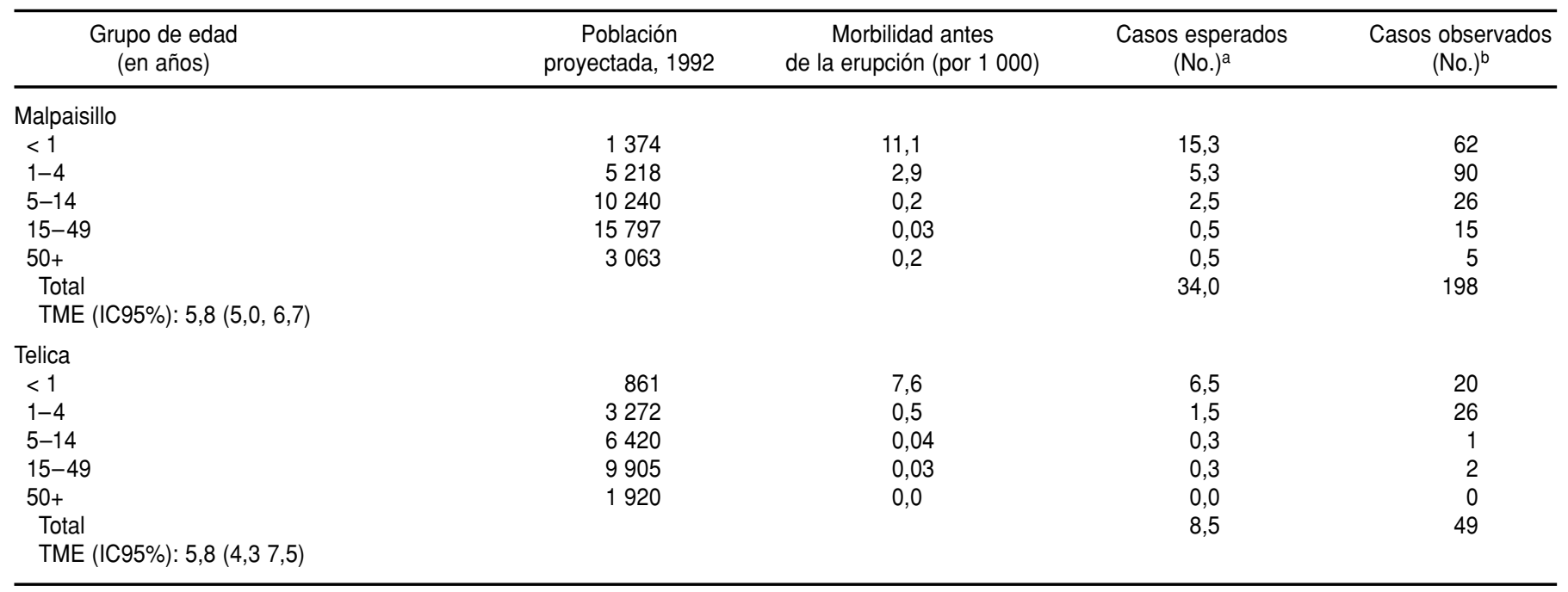

a Se basa en datos del período que abarca desde el 8 de marzo hasta el 4 de abril de 1992.

b 12 a 18 de abril de 1992.

CUADRO 2. Casos de infección respiratoria aguda en personas atendidas en los establecimientos de salud de Malpaisillo y Telica antes y después de la erupción, junto con las tasas de morbilidad estandarizadas (TME) y los intervalos de confianza de $95 \%$ (IC95\%) derivados de esos datos

\begin{tabular}{|c|c|c|c|c|}
\hline $\begin{array}{l}\text { Grupo de edad } \\
\text { (en años) }\end{array}$ & $\begin{array}{c}\text { Población } \\
\text { proyectada, } 1992\end{array}$ & $\begin{array}{c}\text { Morbilidad antes } \\
\text { de la erupción (por } 1000 \text { ) }\end{array}$ & $\begin{array}{l}\text { Casos esperados } \\
\text { (No.) })^{\mathrm{a}}\end{array}$ & 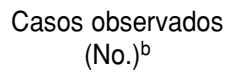 \\
\hline \multicolumn{5}{|l|}{ Malpaisillo } \\
\hline $1-4$ & 5218 & 14,3 & 74,8 & 194 \\
\hline $5-14$ & 10240 & 3,0 & 31,0 & 187 \\
\hline $15-49$ & 15797 & 2,3 & 36,8 & 193 \\
\hline $50+$ & 3063 & 1,4 & 4,3 & 33 \\
\hline \multicolumn{5}{|l|}{ Telica } \\
\hline$<1$ & 861 & 22,9 & 19,8 & 72 \\
\hline $1-4$ & 3272 & 6,4 & 21,0 & 129 \\
\hline $5-14$ & 6420 & 1,5 & 9,8 & 73 \\
\hline $15-49$ & 9905 & 0,7 & 6,8 & 68 \\
\hline $50+$ & 1920 & 0,5 & 1,0 & 10 \\
\hline
\end{tabular}

a Se basa en datos del período que abarca desde el 8 de marzo hasta el 4 de abril de 1992.

b 12 a 18 de abril de 1992.

vuelto a sus casas en esas zonas, puede ser que el número de consultas a servicios de salud después de la erupción haya subrepresentado la morbilidad por diarrea e infecciones respiratorias.

En particular, los residentes de los municipios de Malpaisillo y Telica fueron evacuados a cerca de 20 albergues fuera de la zona afectada, junto con habitantes de León, La Paz Centro, Quezalguaque, Corinto, Chichigalpa y varios lugares situados dentro de un radio de $12 \mathrm{~km}$ de distancia del volcán. Estos residentes fueron trasladados a albergues provisionales en León en los 3 primeros días después de la erupción y al cabo de un día fueron trasladados a campamentos en las cercanías. Las personas evacuadas recibieron atención médica en cuatro puestos de salud que habían sido establecidos dentro de esos campamentos (9).

Aunque las enfermedades gastrointestinales no son causadas directa- 
mente por las erupciones volcánicas, sí han sido notificadas como efectos secundarios del consumo de aguas superficiales sin filtrar y mal cloradas. Se ha observado giardiasis de transmisión hídrica en asociación con las grandes corrientes de agua producidas por acción del clima cálido y de la acumulación de ceniza volcánica en la nieve (13). Se sabe que la acumulación de mucha ceniza también afecta al funcionamiento de las plantas de tratamiento de aguas residuales porque hace rebasar los lechos de filtración, daña la maquinaria y hace que las aguas negras que no han sido tratadas penetren en el agua de superficie (14). En zonas rurales, donde las fuentes de agua principales son los pozos, se pueden crear o exacerbar indirectamente algunas condiciones que predisponen a las enfermedades diarreicas por efecto de la acumulación de ceniza en las fuentes de agua. Estas condiciones, junto con el mal saneamiento y la mala higiene en las zonas rurales, podrían explicar el mayor número de casos de diarrea que se ha observado.

Se han notificado problemas oculares y respiratorios por efecto de la ceniza después de erupciones volcánicas (3, 14-21). Algunos efectos inmediatos han sido la irritación aguda y pasajera de las membranas mucosas de los ojos y de las vías respiratorias por acción de la ceniza y los gases volcánicos y la exacerbación de neumopatías crónicas ya presentes como consecuencia de la gran acumulación de ceniza durante la erupción y el período posterior (15). Una hora después de que empezara la actividad volcánica en Cerro Negro, comenzó a caer la ceniza en León. Al día siguiente los depósitos registrados ya llegaban a 1 $\mathrm{cm}$. Para el 12 de abril, los cambios en la dirección del viento del oeste-sudoeste hacia el oeste (figura 1) causaron acumulaciones de ceniza de aproximadamente 2,5 cm en Telica (22).

En general, la erupción aumentó de dos a 10 veces el número de consultas a servicios de salud por IRA entre los residentes de Malpaisillo y Telica pertenecientes a los grupos de edad estudiados. Las grandes tasas de inciden- cia de IRA observadas en los lactantes de la población estudiada podrían atribuirse a varias circunstancias, incluso a infecciones adquiridas en albergues hacinados que posiblemente fueron tratadas en dispensarios de las zonas de estudio poco después de la erupción, antes de que se establecieran puestos de salud en los asentamientos para personas evacuadas. También es concebible que haya comenzado un nuevo ciclo de infecciones respiratorias. (Después de la erupción del volcán de La Soufrière en la isla de San Vicente en 1979, se observó por primera vez enfermedad respiratoria broncoespástica pasajera en niños menores de un año que antes estaban sanos $(15,23)$.

Antes de la erupción, las tasas semanales de consulta a los establecimientos de atención de salud demostró que la tasa de morbilidad por diarrea e IRA eran mayores en Malpaisillo que en Telica. Después de la erupción se observó un aparente aumento de la incidencia de diarrea e infecciones respiratorias inespecíficas en ambas comunidades; sin embargo, es preciso tener en cuenta los posibles factores de confusión relacionados con las características de las comunidades estudiadas y la cobertura por los centros de salud que están a su servicio.

Atribuimos las mayores tasas de consulta a centros de atención de salud por IRA después de la erupción en Telica, $16 \mathrm{~km}$ al oeste del volcán, a su ubicación en la zona de distribución de la ceniza (figura 1). A diferencia de Telica, el municipio de Malpaisillo está varios kilómetros al norte de Cerro Negro, fuera de la zona donde cayó más ceniza. No obstante, es posible que la mayor tasa de morbilidad por diarrea y por infecciones respiratorias en Malpaisillo, sugerida por las mayores tasas de consulta al centro de salud, se haya debido en parte a la erupción. Puede ser que los centros de salud hayan tratado a personas que vivían dentro de la zona donde cayó la ceniza y también a personas que fueron evacuadas a campamentos vecinos y que recibieron su atención médica fuera de los puestos de salud estableci- dos en esos campamentos. Al mismo tiempo, las instalaciones de salud de Malpaisillo siguieron prestando sus servicios a las comunidades circunvecinas que no fueron afectadas por la erupción y esta población podría haber contribuido al aumento del número de consultas por problemas ajenos a aquella.

Los períodos de comparación idóneos para evaluar el efecto de la erupción hubieran sido los períodos de notificación epidemiológica de años anteriores, particularmente de los últimos 5 años, que fueran idénticos al período de estudio después de la erupción. Sin embargo, no se dispuso de tales datos en el momento de la investigación y, por ende, fue imposible determinar el exceso de la morbilidad. En general, fue difícil determinar, a partir de los datos acopiados, si el mayor número de consultas a servicios de salud por diarrea e infecciones respiratorias que detectó el sistema de vigilancia se debió a los efectos de la erupción en la salud o a un mayor número de consultas por personas que aprovecharon para acudir a los servicios de salud a raíz de ella. Los resultados de esta investigación resaltan, sin embargo, la posible utilidad de los sistemas habituales de información de salud para obtener datos fidedignos sobre la incidencia de enfermedades particulares después de un desastre.

\section{CONCLUSIONES}

Resulta evidente que las erupciones volcánicas pueden tener efectos directos e indirectos, inmediatos y tardíos, en la salud y la seguridad (15). Los efectos de tales fenómenos pueden observarse activamente por medio de los sistemas nacionales de vigilancia que ya están instalados, los cuales proveen información útil sobre las alteraciones del estado de salud de las poblaciones en riesgo. La vigilancia pasiva, como la que aplicó el sistema nacional de vigilancia en el trabajo aquí notificado, puede usarse simultáneamente para suplementar la vigilancia activa cuando hay que observar los 
efectos en la salud en una zona más extensa o por un período más largo.

En vista de los resultados aquí notificados, recomendamos que se investiguen más a fondo los siguientes aspectos:

a) Las enfermedades respiratorias relacionadas con la ceniza. Conviene realizar estudios detallados para evaluar el espectro de tales enfermedades y determinar en qué momento se presentan en subgrupos especiales de la población, como el de los niños menores de un año. b) El tipo y la calidad del abastecimiento de agua en las zonas rurales. El marcado aumento de la incidencia de diarrea aguda después del desastre, particularmente en niños pequeños, exige que se estudie más detalladamente el abastecimiento de agua.

c) El sistema nacional de vigilancia epidemiológica. Este se podría modificar o ampliar a fin de incrementar su sensibilidad a nuevos casos y, por ende, su capacidad para ofrecer servicios apropiados de notificación a los organismos de socorro médico. También se podría modifi- car con el fin de poder examinar los efectos a largo plazo de la exposición a la ceniza en una comunidad en particular.

Agradecimiento. Los autores agradecen el apoyo prestado a su trabajo por Aurora Velásquez y Maritza Montalbán del SILAIS en León, Nicaragua; Stanley Williams de la Universidad del Estado de Arizona, Estados Unidos de América; Peter Baxter de la Universidad de Cambridge, Reino Unido; Claude de Ville de la OPS, Washing-

\section{REFERENCIAS}

1. Thacker SB, Berkelman RL. Public health surveillance in the United States. Epidemiol Rev 1988;10:164-190.

2. Pan American Health Organization. Epidemiologic surveillance after natural disaster. Washington, DC: PAHO; 1982. (Publicación científica 420).

3. Bernstein RS, Baxter PJ, Falk H, Ing R, Foster L, Frost F. Immediate public health concerns and actions in volcanic eruptions: lessons from the Mount St Helen's eruptions, May 18-October 18, 1980. Am J Public Health 1986;76(suppl):25-38.

4. Romero AB, Cobar R, Western KA, Mayorga López S. Some epidemiologic features of disasters in Guatemala. Disasters 1978;2:39-46.

5. Dietz VJ, Rigau-Pérez JG, Sanderson L, Díaz L, Gunn RA. Health assessment of the 1985 flood disaster in Puerto Rico. Disasters 1990;14:164-170.

6. Lee LE, Fonseca V, Brett KM, et al. Active morbidity surveillance after Hurricane Andrew: Florida, 1992. JAMA 1993;270: 591-594.

7. Glass RI, Noji EK. Epidemiologic surveillance following disasters. En: Halperin W, Baker E, Jr., eds. Public health surveillance. New York: Van Nostrand Reinhold; 1992:195-205.

8. Smithsonian Institution. Cerro Negro. Bull Global Volcanism Network 1992;17:7-9.

9. Organización de las Naciones Unidas. Emergencia erupción "Volcán Cerro Negro". Managua: ONU; abril de 1992.
10. Pan American Health Organization. Vol I: Health conditions in the Americas. Edición de 1994. Washington, DC: PAHO; 1994. (Publicación científica 549; 2 vol).

11. Nicaragua, Ministerio de Salud. Census projections, Department of León, Nicaragua. León: MINSA, Sistema Local de Atención Integral en Salud; 1992.

12. Vandenbrouke JP. A shortcut method for calculating the 95 per cent confidence interval of the standardized mortality ratio. Am J Epidemiol 1984;115:303-304.

13. Weniger BG, Blaser MJ, Gedrose J, Lippy EC, Juranek DD. An outbreak of waterborne giardiasis associated with heavy water runoff due to warm weather and volcanic ashfall. Am J Public Health 1983; 8:868-872.

14. Baxter PJ, Bernstein RS, Falk H, French J, Ing R. Medical aspects of volcanic disasters: an outline of the hazards and emergency response measures. Disasters 1982; 6:268-276.

15. Bernstein RS, Baxter PJ, Buist AS. Introduction to the epidemiological aspects of explosive volcanism. Am J Public Health 1986;76:3-9.

16. Seaman J, Hogg C. Volcanoes. En: Seaman J, Lewesley S, Hogg C, eds. Vol 5: Contributions to epidemiology and biostatistics. Basel, Switzerland: S. Karger; 1984: 157-172.

17. Baxter PJ, Ing R, Falk $H$, Plikaytis B. Mount St. Helens eruptions: the acute respiratory effects of volcanic ash in a North
American community. Arch Environ Health 1983;38:138-143.

18. Baxter PJ. Medical effects of volcanic eruptions. Bull Volcanol 1990;52:532-544.

19. Baxter PJ, Ing R, Falk $H$, et al. Mount St. Helens eruptions, May 18 to June 12, 1980: an overview of the acute health impact. JAMA 1981;246:2585-2589.

20. Yano E, Yokoyama Y, Nishii S. Chronic pulmonary effects of volcanic ash: an epidemiologic study. Arch Environ Health 1986;41:94-99.

21. Yano E, Yokoyama Y, Higashi H, Nishii S, Maeda K, Koizumi A. Health effects of volcanic ash: a repeat study. Arch Environ Health 1990;45:367-373.

22. Smithsonian Institution. Cerro Negro. Bull Global Volcanism Network 1992;17:2-3.

23. Leus X, Kintanar C, Bowman V. Asthmatic bronchitis associated with a volcanic eruption in St. Vincent, West Indies [carta]. Disasters 1981;5:67-69.

Manuscrito recibido el 1 de enero de 1996. Aceptado para publicación tras revisión, el 7 de junio de 1996. 
ABSTRACT The eruption of the Cerro Negro volcano near León, Nicaragua, on 9 April 1992 distributed an estimated 1.7 million tons of ash over a 200 square kilometer area. An assessment was conducted to evaluate the health effects on approximately 300000 residents, using routine data obtained by the national epidemiologic surveillance system. It was found that rates of visits to health care facilities for acute diarrheal and respiratory illnesses increased in two study communities, one within and one near the disaster zone. Specifically, visits for acute diarrhea were nearly 6.0 times more numerous than before the eruption in both communities, while visits for acute respiratory diseases were 3.6 times more frequent in Malpaisillo (the community near the disaster zone) and 6.0 times more frequent in Telica (the community within it). Most of the visits were for infants and children less than 5 years old.

Increased diarrheal disease morbidity, which commonly occurs after volcanic eruptions, demands detailed investigation of the type and quality of water supplies following heavy ashfall. Ash-related respiratory problems should be further examined to determine the spectrum of such diseases and the timing of illness onsets among infants and other special population subgroups. Data collected on health conditions before and after an eruption by passive surveillance can be used to detect eruptionrelated morbidity. Systems already in place, such as Nicaragua's national epidemiologic surveillance system, can be modified or extended so as to increase their sensitivity to new cases and hence their ability to provide appropriate notification to medical relief agencies.

Public health surveillance Nicaragua, 1992

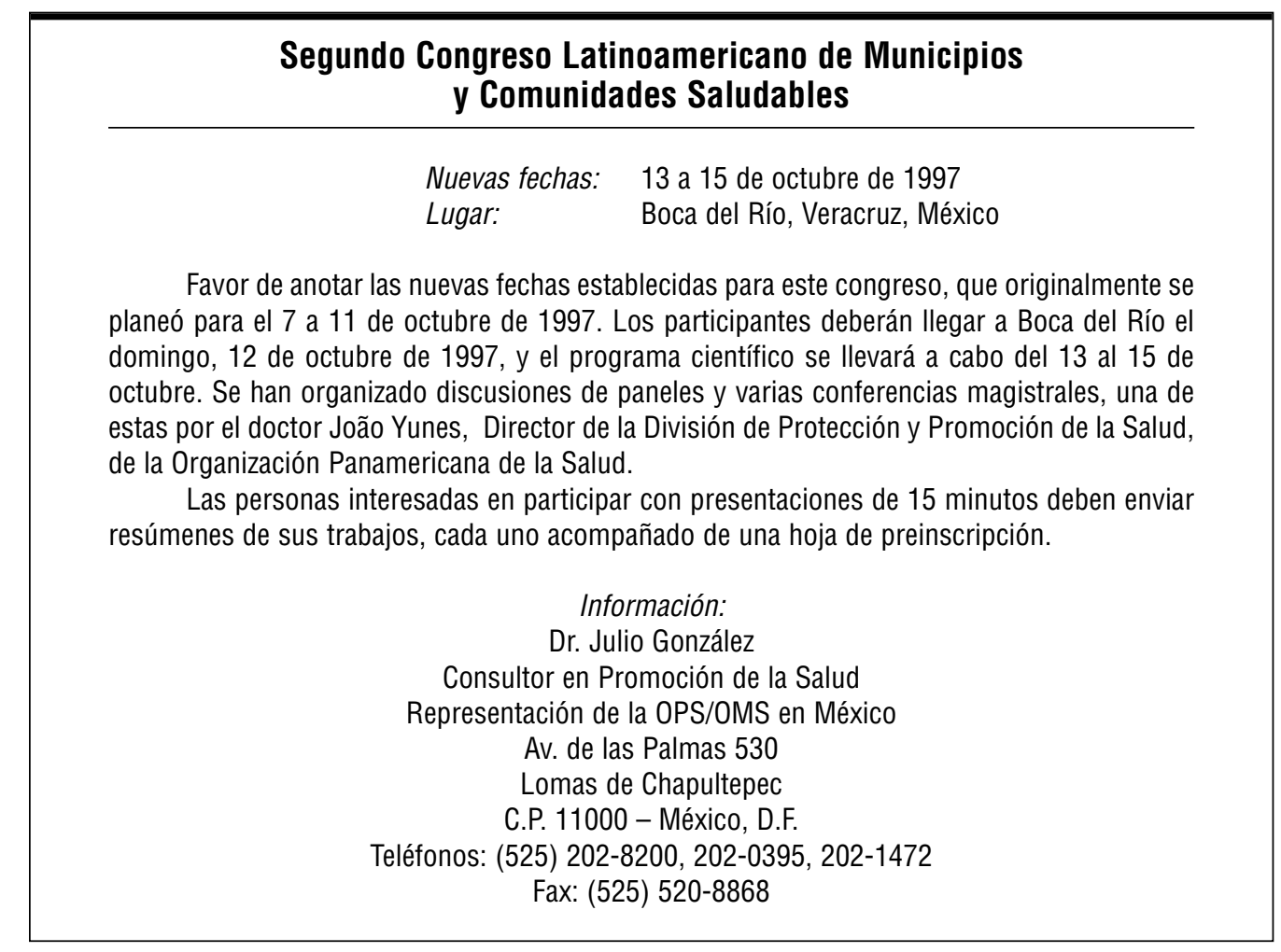

